International Journal of Pure and Applied Mathematics

Volume 102 No. 2 2015, 209-223

ISSN: 1311-8080 (printed version); ISSN: 1314-3395 (on-line version)

url: http://www.ijpam.eu

doi: http://dx.doi.org/10.12732/ijpam.v102i2.4

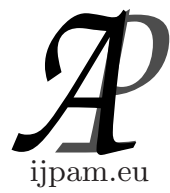

\title{
CONTROL OF EPIDEMIC BY VACCINATION IN \\ A SIRS MODEL WITH TWO INFECTED CATEGORIES
}

\author{
M. Baniasadi Moghadam ${ }^{1}$, O. Rabiei Motlagh², H.M. Mohamadi Nezhad ${ }^{3}$ \\ 1,2,3 Department of Mathematics \\ University of Birjand \\ Birjand, IRAN
}

\begin{abstract}
The aim of this paper is investigating the effect of vaccinating on the behavior of an infectious disease by study the effect on the basic reproduction number $\left(R_{0}(p)\right)$, in a population where two different categories of infected (also susceptible) individuals exist, the differences between these two infected (also susceptible) categories are the rates of infection transmission and recovery (for susceptible incidence rate) and vaccination program is carrying out by vaccinating two different percentages of these two different categories of susceptible individuals. To study the behavior of infectious disease $R_{0}(p)$, is computed and the relationship between $R_{0}(p)$ and the existence, stability and bifurcation of equilibriums is investigated. Also conditions that lead to removing the infection are obtained.
\end{abstract}

AMS Subject Classification: 92D25, 92D30

Key Words: basic reproduction number, two stages SIR models, vaccination program, backward bifurcation, stability

\section{Introduction}

Population growth and spread, global climate change, and the emergence and

Received: January 20, 2015

(C) 2015 Academic Publications, Ltd.

${ }^{\S}$ Correspondence author url: www.acadpubl.eu 
reemergence of novel and deadly forms of infectious diseases have increased the need for sound quantitative methods to guide disease intervention practice $[1,7,10]$. In the 20th century, influenza was pandemic several times and new diseases such as Lyme disease, Legionnaire's disease, toxic-shock syndrome, hepatitis C, hepatitis E, and hantavirus were encountered. The human immunodeficiency virus (HIV), which is the etiologic agent for acquired immunodeficiency syndrome (AIDS), was identified in 1981 and now causes over 3 million deaths per year in the world. Drug and antibiotic resistance have become serious issues for diseases such as tuberculosis, malaria, and gonorrhea. Prion have been identified as the infectious agents for bovine spongiform encephalopathy (BSE or mad-cow disease), Creutzfeldt-jacob disease, kuru, and scrapie in sheep. Changing patterns of social behavior and travel present new classes of disease transmission problems. For example, west Nile virus has spread to north America. Biological terrorism with diseases such as smallpox or plague has become a new threat. In the 21st century, we have already encountered severe acute respiratory syndrome (SARS), now we are engaging with Ebola disease and will undoubtedly face more new infectious disease challenges.

The epidemiological modeling of infectious disease transmission has a long history in mathematical biology, but in recent years it has had an increasing influence on the theory and practice of disease management and control $[7,8,11]$. Mathematical modeling of the spread of infectious diseases has become part of epidemiology policy decision making in several countries. Epidemiological modeling studies of diseases such as gonorrhea, HIV/AIDS, BSE, foot and mouth disease, measles, rubella, and pertussis have had an impact on public health policy in these countries. Thus modeling approaches have become very important for decision-making about infectious disease intervention programs. Recent approaches include deterministic models, computer simulation, Markov chain, Monto Carlo models, small world and other network models, stochastic models, and microsimulation of individuals in a community. These techniques are often implemented computationally and use data on disease incidence and population demographics. Sometimes the epidemiology, immunology, and evolution of a disease must all be considered. For example, some recent research has studied the rational design of influenza vaccines by considering the effects on the immunology of influenza immunity in individuals of the yearly epidemics of influenza A variants, the vaccine composition each year, and the yearly evolutionary drift of influenza A virus variants [2].

In studying the behavior of an infectious disease, one quantity stands out, the ratio of the rate of infection to the rate of recovery or equivalently, the average number of new cases produced when one infected individual is introduced 
into a completely susceptible community. This quantity is called the reproduction number and it is shown by $R_{0}$, it has been shown that $R_{0}$ is mathematically characterized by regarding infection transmission as a demographic process, where producing offspring is not seen as giving birth in the demographic sense, but as causing a new infection through transmission. In a natural way this leads to viewing the infection process in terms of consecutive generations of infected individuals, in complete analogy to demographic generation. Subsequent generations growing in size then indicate a growing population, and the growth factor per generation indicates the potential for growth. In a natural way this growth factor is then the mathematical characterization of $R_{0}[4,5]$. By this point of view recently in $[5,6]$ a new method for computing $R_{0}$ is presented. Always a threshold value for the basic reproduction number determine whether an epidemic outbreak follows the introduction of an infected individual into a completely susceptible community or not, more precisely if $R_{0}$ is more than threshold value introduction of an infected individuals may leads to epidemic out break and if $R_{0}$ is less than it epidemic outbreak does not happen.

In [12] the authors have supposed an infectious disease have infected a community with constant size with this assumption: primary infected (the individuals that being infected for the first time) and secondary infected (the individuals which having experienced the infection at least once before) are recovered and transmit the infection in different rates also they have supposed the infection has not vertical transmission, so newborns are primary susceptible, to control the infection they have considered a vaccination program such that a constant percentage of newborns are vaccinated, the aim of [12] is investigating the program success.

In [3] the authors have concentrated on a more generally situation that involve [12] as an special case in this way: differences between primary and secondary infected or susceptible individuals, the size of population and vertical transmission are the same as [12], but to control the infectious disease a vaccination program is done such that a constant percentage of primary are vaccinated, obviously the vaccination program in [12] is obtained by limiting the vaccination in [3] to newborns. In this work we generalize the conditions of infection and vaccination to include [3] as an special case. We give a brief outline of the work: A nonfatal infectious disease is linearly spreading in a community with constant size ( the incidence rate is proportional to the density of infected and susceptible individuals), such that the acquired immunity vanishes as time goes on and there is vertical transmission (almost every infection, such as HIV, Hepatitis, Syphilis, Toxoplasmosis, Cytomegalovirus, Rubella, Listeriosis has vertical transmission therefore this paper is a good supplement to [3]), 
the rates of infection transmission and recovery are different in primary and secondary infected individuals and the rate of incidence is different in primary and secondary susceptible individuals. A vaccination program is carrying out to control the infection such that a percentage of primary and a different percentage of secondary susceptible individuals are vaccinated simultaneously [in this point of view this work is a supplement for [3]]. In this paper we answer to these important questions by the help of the notion of reproduction number, $R_{0}(p)$ as follows:

Question: What is the necessary condition to start the program?

Answer: $R_{0}(1)<1<R_{0}(0)$ is sufficient and necessary to start the program.

Question: What percentages of susceptible categories must be vaccinated to have a successful program?

Answer: $p_{1}$ percent of primary susceptible individuals and $g p_{1}$ percent of secondary susceptible individuals must be vaccinated for a successful program, if $p$ increases the infection damping process is faster ( $p_{1}$ will be introduced).

Question: How much we can increase vaccination coverage?

Answer: we can extend the vaccination to the whole of population.

Question: When the infection removes?

Answer: $R_{0}(p)<1$ with another condition related to parameters implies that the infection will be removed, we guess $R_{0}(p)<1$ is singly sufficient for it, we have investigated this guess by numerical simulation, if $R_{0}(p)>1$ an endemic or epidemic may happen.

\section{Dimensionless Model and $\boldsymbol{R}_{o}(p)$}

Consider a population with constant size and an infection in it such that: there is difference between individuals being infected for the first time (primary infected) and individuals having experienced the infection at least once before (secondary infected). Hence the total population is subdivided into: individuals who have never experienced the infection(primary susceptible, whose population is $S_{1}$ ), primarily infected individuals (whose population is $I_{1}$ ), recovered individuals (whose population is $R$ ), individuals who have lost their acquired immunity (secondary susceptible, whose population is $S_{2}$ ), secondary infected individuals (whose population is $I_{2}$ ) and vaccinated individuals (whose 
population is $V$ ). If we show the total population by $N(t)$ and suppose homogenous mixing of population we have: at time " $t$ " a parturient with probabilities $\left(I_{1}(t)+I_{2}(t)\right) / N(t),\left(S_{1}(t)+S_{2}(t)\right) / N(t)$ and $(V(t)+R(t)) / N(t)$ respectively is infected, susceptible and immune. By vertical transmission, antibody and antigen are transferred from mothers to fetuses therefore infected, susceptible and immune mothers respectively have $I_{1}, S_{1}$ and $V$ neonates, if we assume that individuals to be born with rate $\mu$, the portion of $S_{1}, I_{1}$ and $V$ of newborns respectively is $\mu\left(S_{1}+S_{2}\right), \mu\left(I_{1}+I_{2}\right)$ and $\mu(R+V)$. Also we suppose that individuals in $S_{1}$ die with rate $\mu$ and get infected with primary rate of infection $\lambda(t)=k\left(I_{1}+r I_{2}\right)$, where $k$ is the transmission rate of $I_{1}$ and $r$ is the relative infectivity of $I_{2}$ with respect to $I_{1}$, also they get vaccinated with rate $p$. Individuals in $I_{1}$ die with rate $\mu$ (the infection is not fatal) and recover with rate $\alpha$. Individuals in $R$ die with rate $\mu$ and lose their immunity with rate $\sigma$, to become $S_{2}$. Individuals in $S_{2}$ die with rate $\mu$ and get infected with secondary rate of infection $g \lambda(t)$, where $g$ represents the relative susceptibility of $S_{2}$ with respect to $S_{1}$ and get vaccinated with rate $g p$. Individuals in $I_{2}$ die with rate $\mu$ (the infection is not fatal) and recover with rate $q \alpha$ where $q$ represents the relation of the recovery rate of $I_{2}$ to the recovery rate of $I_{1}$ and finally individuals in $V$ die with rate $\mu$ and lose their immunity and become $S_{2}$ with rate $b \sigma$ the parameter $b$ represents the relative loss of immunity of $V$ with respect to $R$. If $b=0$, then the vaccine conveys life long protection, while a value of $b=1$ means that the vaccine induced immunity lasts as long as natural immunity. All parameters $r, b, g$ and $q$ are dimensionless. If $r>1$, then $I_{2}$ have higher transmissibility than $I_{1}$ and vice versa. However, If $q>1$, then the infectious period of $I_{2}$ is shorter than that of $I_{1}$ and vice versa. Finally, a value of $g<1$ means that $S_{2}$ have reduced susceptibility and vice versa. We restrict ourselves to the case $g<1$. If we show the proportion of subpopulation i.e. $I_{1}\left(t / \mu_{0}\right) / N$, $I_{2}\left(t / \mu_{0}\right) / N, S_{1}\left(t / \mu_{0}\right) / N, R\left(t / \mu_{0}\right) / N, V\left(t / \mu_{0}\right) / N$ and $S_{2}\left(t / \mu_{0}\right) / N$ (where $\mu_{0}$ is a quantity with dimension "time" and value "1") again by $I_{1}, I_{2}, S_{1}, R, V$ and $S_{2}$ then we have the following dimension less system

$$
\begin{aligned}
\frac{d}{d t} S_{1} & =\mu S_{2}-(p+\lambda(t)) S_{1} \\
\frac{d}{d t} S_{2} & =b \sigma V+\sigma R-(g p+\mu+g \lambda(t)) S_{2} \\
\frac{d}{d t} I_{1} & =\lambda(t) S_{1}+\mu I_{2}-\alpha I_{1} \\
\frac{d}{d t} I_{2} & =g \lambda(t) S_{2}-(\mu+q \alpha) I_{2}
\end{aligned}
$$




$$
\begin{aligned}
\frac{d}{d t} R & =\alpha I_{1}+q \alpha I_{2}-(\sigma+\mu) R \\
\frac{d}{d t} V & =p S_{1}+\mu R+g p S_{2}-b \sigma V
\end{aligned}
$$

$\lambda(t)=\kappa\left(I_{1}+r I_{2}\right)$ where $\kappa=N k$. Clearly $S_{1}+S_{2}+I_{1}+I_{2}+R+V=1$ all parameters and all subpopulation proportions are nonnegative. The solutions of system (1) exist and remain nonnegative for nonnegative initial conditions.

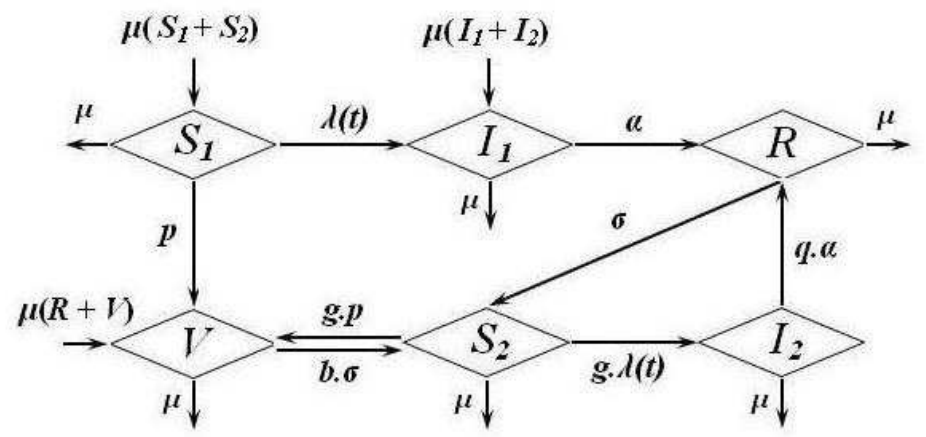

Figure. 1 compartmental model to show the transitions between model states

The basic reproduction number denoted by $R_{0}$, is the average number of new cases produced by an infected case, during the infection period, when introduced into a infection free population with a vaccination program [9]. Model (1) has the infection free equilibrium

$$
\begin{array}{r}
E_{0}=\left(S_{1}^{0}, S_{2}^{0}, I_{1}^{0}, I_{2}^{0}, R^{0}, V^{0}\right)=\left(\frac{b \mu \sigma}{b \mu \sigma+p(\mu+b \sigma+g p)}, \frac{b p \sigma}{b \mu \sigma+p(\mu+b \sigma+g p)}\right. \\
\left.0,0,0, \frac{p(\mu+g p)}{b \mu \sigma+p(\mu+b \sigma+g p)}\right)
\end{array}
$$

based on the general approach in $[5,6]$ we have $T$ and $\Sigma$ as

$$
T=\left(\begin{array}{lr}
\kappa S_{1}^{0} & r \kappa S_{1}^{0} \\
g \kappa S_{2}^{0} & r g \kappa S_{2}^{0}
\end{array}\right), \quad \Sigma=\left(\begin{array}{lr}
-\alpha & \mu \\
0 & -(q \alpha+\mu)
\end{array}\right) .
$$

hence $K_{L}$ is given by

$$
K_{L}=-T \Sigma^{-1}=\left(\begin{array}{lr}
\kappa S_{1}^{0} & r \kappa S_{1}^{0} \\
g \kappa S_{2}^{0} & r g \kappa S_{2}^{0}
\end{array}\right)\left(\begin{array}{lr}
1 / \alpha & \mu / \alpha(q \alpha+\mu) \\
0 & 1 /(q \alpha+\mu)
\end{array}\right)
$$




$$
=\left(\begin{array}{ll}
\kappa S_{1}^{0} / \alpha & \kappa S_{1}^{0}((\mu / \alpha)+r) /(q \alpha+\mu) \\
g \kappa S_{2}^{0} / \alpha & g \kappa S_{2}^{0}((\mu / \alpha)+r) /(q \alpha+\mu)
\end{array}\right) .
$$

so $R_{0}$, is given by

$$
R_{0}(p)=\rho\left(K_{L}\right)=\frac{b \kappa \sigma(\mu(q \alpha+\mu)+g p(r \alpha+\mu))}{\alpha(q \alpha+\mu)(p(g p+\mu)+b \sigma(p+\mu))}
$$

In this work $R_{0}(p)$, plays an important role. In section (3) we show that $R_{0}(p)<$ 1 with condition (6) imply the global stability of $E_{0}$ (infection free equilibrium), which grantees the program success, in section (4) we obtain necessary condition to start the vaccinating and a threshold $p_{1}$, such that if $p>p_{1}$, then $R_{0}(p)<$ 1 , and finally by numerical simulation we conclude that $R_{0}(p)<1$, singly is sufficient for global stability of $E_{0}$, also $R_{0}(p)>1$, is sufficient for existence of $E_{1}$ (endemic equilibrium), so the model has backward bifurcation in $p_{1}$. We have the following main results.

\section{Main Results}

Consider the two stages SIRS model (1), we have:

(1) The necessary condition to start vaccinating is: $R_{0}(1)<1<R_{0}(0)$.

(2) To have a successful program we must vaccine at least $p_{1}$, percentage of $S_{1}$ and $g p_{1}$, percentage of $S_{2}$, also we can increase $p$ to 1 .

(3) $R_{0}(p)<1$, and condition (6) imply the global stability of $E_{0}$ which grantees removing the infection.

(4) If $p<p_{1}$, system has two equilibriums and if $p>p_{1}$, it has one equilibrium so in $p_{1}$, model has a backward bifurcation.

\section{Existence and Stability of $E_{0}$}

In this section the relationship between $R_{0}(p)$, and stability of $E_{0}$ is studied. Model (1) is reduced to a model with lower dimension since the size of population is constant, as follows:

$$
\begin{aligned}
\frac{d}{d t} S_{1} & =\mu S_{2}-(p+\lambda(t)) S_{1} \\
\frac{d}{d t} S_{2} & =b \sigma-b \sigma I_{1}-b \sigma I_{2}-b \sigma S_{1}-\sigma(b-1) R-(g \lambda(t)+\mu+g p+b \sigma) S_{2}
\end{aligned}
$$




$$
\begin{aligned}
\frac{d}{d t} I_{1} & =\lambda(t) S_{1}+\mu I_{2}-\alpha I_{1} \\
\frac{d}{d t} I_{2} & =g \lambda(t) S_{2}-(\mu+q \alpha) I_{2} \\
\frac{d}{d t} R & =\alpha\left(I_{1}+q I_{2}\right)-(\sigma+\mu) R
\end{aligned}
$$

we study (4) in the following region

$$
\Delta=\left\{\left(S_{1}, S_{2}, I_{1}, I_{2}, R\right): S_{1}, S_{2}, I_{1}, I_{2}, R \geq 0, S_{1}+S_{2}+I_{1}+I_{2}+R \leq 1\right\}
$$

if $J_{0}=(b \mu \sigma /(b \mu \sigma+p(g p+\mu+b \sigma)), b p \sigma /(b \mu \sigma+p(g p+\mu+b \sigma)), 0,0,0)$.

The linear part of $(4)$ at $J_{0}$ is:

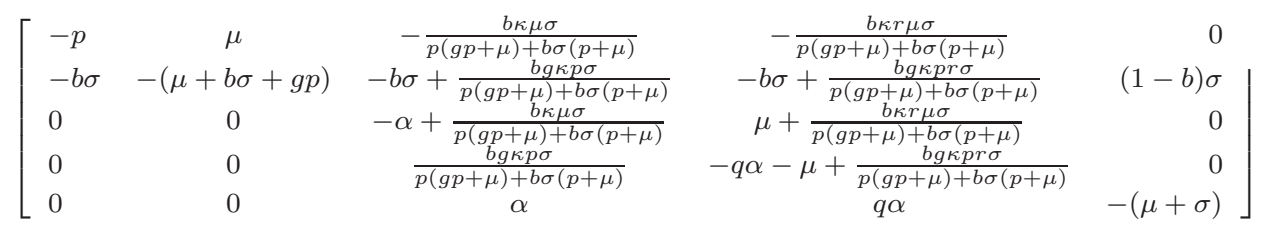

The eigenvalues are $x_{1}=-(\sigma+\mu)<0, x_{2}, x_{3}$, (roots of the following equation)

$$
\begin{gathered}
x^{2}+\xi_{1} x+\zeta_{1}=0 \\
\xi_{1}=b \sigma+p+g p+\mu \\
\zeta_{1}=b p \sigma+g p^{2}+b \mu \sigma+p \mu
\end{gathered}
$$

since all of coefficients are positive the real parts of $x_{2}$ and $x_{3}$ are negative, and $x_{4}, x_{5}$, (roots of the following equation)

$$
\psi_{2} x^{2}+\xi_{2} x+\zeta_{2}=0
$$

$$
\begin{aligned}
\psi_{2} & =g p^{2}+p \mu+b p \sigma+b \mu \sigma \\
\xi_{2} & =g p^{2} \alpha+g p^{2} q \alpha+g p^{2} \mu+p \alpha \mu+p q \alpha \mu+p \mu^{2}-b g p r \kappa \sigma+b p \alpha \sigma+b p q \alpha \sigma \\
& -b \kappa \mu \sigma+b p \mu \sigma+b \alpha \mu \sigma+b q \alpha \mu \sigma+b \mu^{2} \sigma \\
\zeta_{2} & =g q p^{2} \alpha^{2}+g p^{2} \alpha \mu+p q \mu \alpha^{2}+p \alpha \mu^{2}-b g p r \alpha \sigma \kappa+b p q \sigma \alpha^{2}-b g p \kappa \mu \sigma \\
& +b p \alpha \mu \sigma-b q \kappa \alpha \mu \sigma+b q \alpha^{2} \mu \sigma-b \kappa \mu^{2} \sigma+b \alpha \mu^{2} \sigma .
\end{aligned}
$$

Since $\psi_{2}$ is positive, if $\xi_{2}$ and $\zeta_{2}$ are positive then real parts of $x_{4}$ and $x_{5}$ are negative and if $\psi_{2}$ is negative then real part of $x_{4}$ or $x_{5}$ is positive. We have:

$$
R_{0}(p)-1<\frac{\mu+g p \mu^{2}+g p \alpha \mu(1+q)+\alpha(\operatorname{gpr} \alpha+q+2)}{\alpha(g p r(q \alpha+\mu)+1)} \Leftrightarrow \xi_{2}>0
$$




$$
R_{0}(p)-1<0 \Leftrightarrow \zeta_{2}>0
$$

therefore the local stability of $E_{0}$, is determined by the following proposition.

Proposition 3.1. $\quad E_{0}$ exists for all parameter values and is locally stable if and only if $R_{0}(p)<1$.

Obviously $\left(S_{1}, S_{2}, R\right)$ space is an invariant manifold for model (4). By projecting (4) on this space, we have the following system.

$$
\begin{aligned}
\frac{d}{d t} S_{1} & =\mu S_{2}-p S_{1} \\
\frac{d}{d t} S_{2} & =-b \sigma S_{1}-(g p+b \sigma+\mu) S_{2}-\sigma(b-1) R+b \sigma \\
\frac{d}{d t} R & =-(\sigma+\mu) R
\end{aligned}
$$

(5) is linear with only one equilibrium $\left(S_{1}^{0}, S_{2}^{0}, R^{0}\right)$, the eigenvalues at equilibrium are $x_{1}=-(\sigma+\mu), x_{2}, x_{3}$, the roots of the equation

$$
\begin{gathered}
x^{2}+\xi_{3} x+\zeta_{3}=0 \\
\xi_{3}=b \sigma+\mu+g p+p \\
\zeta_{3}=b p \sigma+b \sigma \mu+p \mu+g p^{2} .
\end{gathered}
$$

Similarly, every coefficients are positive so real part of eigenvalues are negative, hence $\left(S_{1}^{0}, S_{2}^{0}, R^{0}\right)$ is stable so, every solution of (5), converges to it. In other word every solution of (4), that is contained entirely in $\left(S_{1}, S_{2}, R\right)$ space, converges to $J_{0}$. If we can obtain conditions that, every solutions of (4) approaches $\left(S_{1}, S_{2}, R\right)$ space, by the continuity with respect to the initial values, we conclude that every solution of (4), converges to $J_{0}$, therefore $j_{0}$ is globally stable. If we suppose

$$
\alpha>\kappa(1+g) \max \left(1, \frac{r}{q}\right)
$$

we have

$$
\frac{d\left(I_{1}+I_{2}\right)}{d t}=I_{1}\left(\kappa S_{1}-\alpha+g \kappa S_{2}\right)+I_{2}\left(r \kappa S_{1}+r g \kappa S_{2}-q \alpha\right)<0
$$

if $\eta(t)=\left(S_{1}(t), S_{2}(t), I_{1}(t), I_{2}(t), R(t)\right)$ is a solution of (4), then $I_{1}(t)+I_{2}(t)$ converges to zero as $t \rightarrow \infty$, therefore $\eta(t)$ approaches $\left(S_{1}, S_{2}, R\right)$ space. So the global stability of $E_{0}$ is determined by the following proposition. 
Proposition 3.2. If $R_{0}(p)<1$ and (6) is satisfied then the infection free equilibrium is globally stable. So part (3) of main results is satisfied.

Conjecture 3.3. we guess $R_{0}(p)<1$ is sufficient for global stability of $E_{0}$, and $R_{0}(p)>1$ implies that there exist one endemic equilibrium $E_{1}$, but we can not present sufficient mathematical reasons, numerical simulation confirm the guess (see section (4)).

Remark 3.4. By proposition (3.1) if $R_{0}(p)>1, E_{0}$ is unstable so the maximum dimension of it's stable manifold is 4 , since every four dimensional submanifold of $\mathbb{R}^{5}$ has lebesgue measure zero, almost every solution of (4) in a neighborhood of $E_{0}$ doesn't belong to it's stable manifold, in other word, almost every solution of (4) in a neighborhood of $E_{o}$ repelled from a neighborhood of $E_{0}$ so, if $R_{0}(p)>1$ introducing even few infected individuals into a infection free population with vaccination program might bring it into a pandemic.

\section{A threshold for percentage of vaccination, Numerically investigation of global stability of $E_{0}$ and backward bifurcation}

In this section firstly necessary condition to start vaccinating, secondly a threshold for vaccine coverage percentage, $p_{1}$, to avoiding epidemic when a completely susceptible community encounter few infected individual is obtained and finally by numerical computation validity of conjecture (3.3) is investigated. If $R_{0}(0)<1$ by proposition (3.2) and conjecture (3.3) vaccination isn't necessary, then we suppose $R_{0}(0)>1$, now we are looking for a $p$ such that $R_{0}(p)<1$, by (3) we have $R_{0}(p)>1,=1,<1$ respectively equivalent to

$$
\begin{gathered}
\psi p^{2}+\xi p+\zeta<0,=0,>0 \\
\psi=g q \alpha^{2}+g \alpha \mu \\
\xi=q \alpha^{2} \mu+b q \sigma \alpha^{2}+\alpha \mu^{2}+b \alpha \mu \sigma-b g r \kappa \alpha \sigma-b g \kappa \mu \sigma \\
\zeta=b q \alpha^{2} \mu \sigma+b \alpha \mu^{2} \sigma-b \kappa \mu^{2} \sigma-b q \kappa \alpha \mu \sigma
\end{gathered}
$$

Since $\psi>o$ then $\psi p^{2}+\xi p+\zeta$ is positive when $p$ is out of it's roots, it's roots is shown by $p_{0}<p_{1}$, certainly $p_{0}<0$, otherwise 0 is out of roots and $R_{0}(0)<1$ which is contrary, hence $(7)$ has at most one root in $(0,1)$, if $p_{1} \in(0,1)$ for every $p \in\left(p_{1}, 1\right), \frac{\partial}{\partial p} R_{0}(p)<0$, so $R_{0}(p)$ is decreasing on $\left(p_{1}, 1\right)$, then certainly 
$R_{0}(1)<1$, vice versa if $R_{0}(1)<1$, obviously there exist a $p$ such that $R_{0}(p)<1$. Therefore the necessary condition to start vaccinating is $R_{0}(1)<1<R_{0}(0)$ and if we start vaccinating $p$ should be greater than $p_{1}$, also increasing $p$ makes infection damping process faster.

Proposition 4.1. Necessary condition to start vaccinating is: $R_{0}(1)<1<$ $R_{0}(0)$, and we should vaccine at least $p_{1}$ percent of $S_{1}$ and gp percent of $S_{2}$. Parts (1) and (2) of main results are satisfied.

For conjecture (3.3) in table (1) we correspond numerical values to parameters of model (4), in each row the necessary condition for vaccinating is satisfied, we see $R_{0}(p)>1$ if and only if there is an endemic point, this is one part of conjecture, hence model (4) has backward bifurcation in $p_{1}$ (see table1). For global stability of $E_{0}$, we again correspond numerical values to parameters such that, $R_{0}(p)<1$ and (6) is't satisfied. If we set the parameters in the 4 th row of table withe $p=0.4$ in (4) then necessary condition for vaccination is satisfied, condition (6) is not satisfied and the following system is obtained

$$
\begin{aligned}
\frac{d}{d t} S_{1} & =0.1 S_{2}-0.4 S_{1}-0.9 I_{1} S_{1}-1.71 I_{2} S_{1} \\
\frac{d}{d t} S_{2} & =-0.06 I_{1}-0.62 I_{2}-0.06 S_{1}+0.05 R \\
& -0.67 I_{1} S_{2}-2.44 I_{2} S_{2}-0.46 S_{2}+0.06 \\
\frac{d}{d t} I_{1} & =0.9 I_{1} S_{1}+1.7 I_{2} S_{1}+0.1 I_{2}-0.2 I_{1} \\
\frac{d}{d t} I_{2} & =0.67 I_{1} S_{1}+2.44 I_{2} S_{1}+0.42 I_{2} \\
\frac{d}{d t} R & =0.2 I_{1}+0.22 I_{2}-0.35 R
\end{aligned}
$$

with reference to table $E_{0}=(0.033,0.131,0,0,0)$, for global stability of $E_{0}$, a number of solutions of (8) with the following initial values are computed and for these solutions the graph of each component is plotted in a figure. (see figures $2,3,4,5,6)$

$$
\begin{aligned}
& \left(S_{1}(0), S_{2}(0), I_{1}(0), I_{2}(0), R(0)\right)=(0.048,0.023,0.097,0.014,0.159) \\
& \left(S_{1}(0), S_{2}(0), I_{1}(0), I_{2}(0), R(0)\right)=(0.140,0.178,0.183,0.438,0.199) \\
& \left(S_{1}(0), S_{2}(0), I_{1}(0), I_{2}(0), R(0)\right)=(0.183,0.120,0.112,0.177,0.137) \\
& \left(S_{1}(0), S_{2}(0), I_{1}(0), I_{2}(0), R(0)\right)=(0.084,0.110,0.011,0.095,0.145) \\
& \left(S_{1}(0), S_{2}(0), I_{1}(0), I_{2}(0), R(0)\right)=(0.177,0.222,0.028,0.158,0.158)
\end{aligned}
$$


as figures $[2,3,4,5]$ show every solution goes to $E_{0}$.

Remark 4.2. By numerical simulation, (4) has a backward bifurcation in $p=p_{1}$, if $p>p_{1}$ it has only one equilibrium $E_{0}$, and if $p<p_{1}$ it has two equilibriums $E_{0}, E_{1}$. Also numerical simulation shows $R_{0}(p)<1$ is sufficient for global stability of $E_{0}$. Thus part (4) of main results is satisfied.

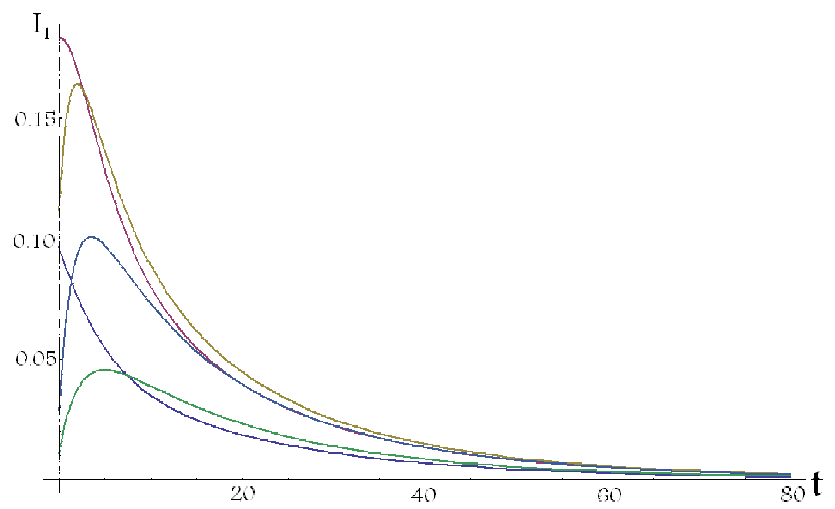

Figure 2. $\lim I_{1}(t)_{t \rightarrow \infty}=0$

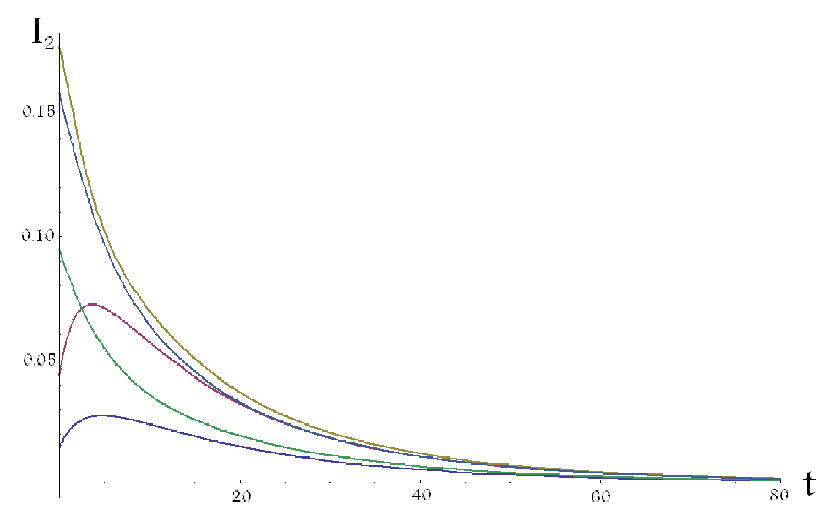

Figure 3. $\lim I_{2}(t)_{t \rightarrow \infty}=0$ 


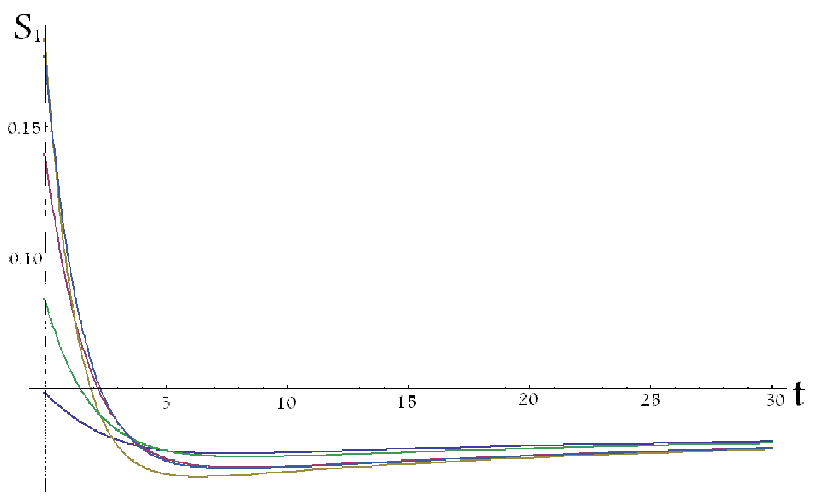

Figure 4. $\lim S_{1}(t)_{t \rightarrow \infty}=0.033$

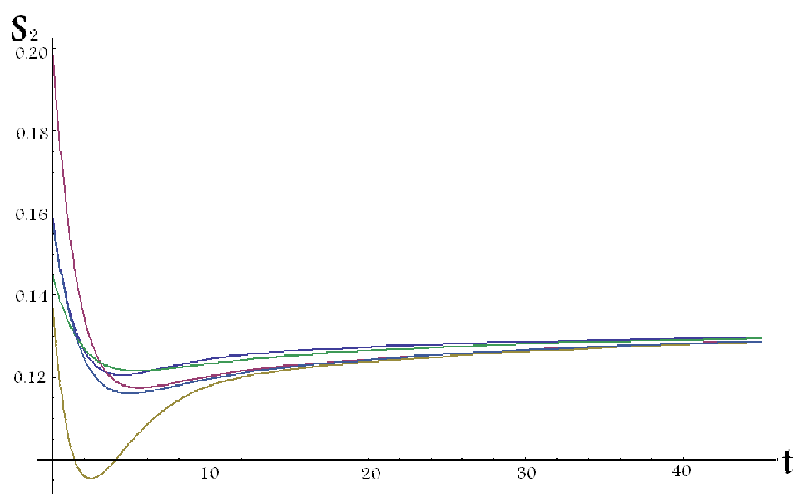

Figure 5. $\lim S_{2}(t)_{t \rightarrow \infty}=0.131$

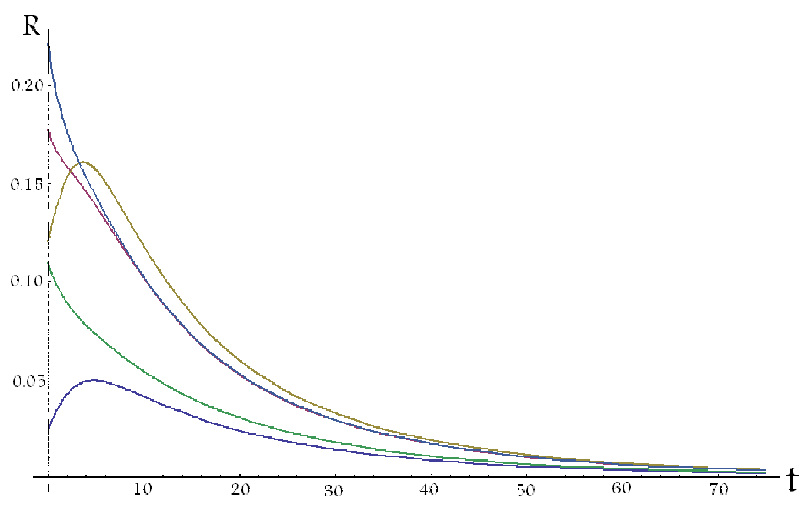

Figure 6. $\lim R(t)_{t \rightarrow \infty}=0$ 


\begin{tabular}{|c|c|c|c|}
\hline Parameters & $\begin{array}{l}\text { Minimum } \\
\text { vaccine cov- } \\
\text { erage }\end{array}$ & $\begin{array}{l}\text { Vaccine coverage } \\
\text { Reproduction num- } \\
\text { ber }\end{array}$ & Equilibrium(s) \\
\hline \multirow{2}{*}{$\begin{array}{l}b=0.5 \kappa=0.15 \\
g=0.2 \mu=0.05 \\
q=0.5 \alpha=0.1 \\
r=0.1 \quad \sigma=0.85\end{array}$} & \multirow[t]{2}{*}{$P_{1}=0.011$} & $\begin{array}{l}P=0.01 \\
R_{0}(P)=1.085\end{array}$ & $\begin{array}{l}E_{0}=(0,0,0.370,0,0.370) \\
E_{1}=(0.001,0,0.338,0.027,0.376)\end{array}$ \\
\hline & & $\begin{array}{l}P=0.012 \\
R_{0}(P)=0.966\end{array}$ & $\begin{array}{l}E_{0}=(0,0,0.324,0,0.389) \\
E_{1}: \text { Doesn't exist }\end{array}$ \\
\hline \multirow{2}{*}{$\begin{array}{l}b=0.5 \kappa=0.15 \\
g=0.2 \mu=0.05 \\
q=0.5 \alpha=0.1 \\
r=0.1 \quad \sigma=0.85\end{array}$} & \multirow[t]{2}{*}{$P_{1}=0.026$} & $\begin{array}{l}P=0 \\
R_{0}(P)=1.5\end{array}$ & $\begin{array}{l}E_{0}=(0,0,1,0,0) \\
E_{1}=(0.115,0.008,0.640,0.0132,0.222)\end{array}$ \\
\hline & & $\begin{array}{l}P=0.1 \\
R_{0}(P)=0.559\end{array}$ & $\begin{array}{l}E_{0}=(0,0,0.300,0,0.600) \\
E_{1}: \text { Doesn't exist }\end{array}$ \\
\hline \multirow{2}{*}{$\begin{array}{l}b=1.5 \kappa=2 \\
g=0.9 \mu=0.9 \\
q=1.5 \alpha=0.9 \\
r=0.05 \sigma=0.6\end{array}$} & \multirow[t]{2}{*}{$P_{1}=0.618$} & $\begin{array}{l}P=0.5 \\
R_{0}(P)=1.126\end{array}$ & $\begin{array}{l}E_{0}=(0,0,0.419,0,0.232) \\
E_{1}=(0.033,0.006,0.363,0.026,0.229)\end{array}$ \\
\hline & & $\begin{array}{l}P=0.7 \\
R_{0}(P)=0.928\end{array}$ & $\begin{array}{l}E_{0}=(0,0,0.323,0,0.251) \\
E_{1}: \text { Doesn't exist }\end{array}$ \\
\hline \multirow{2}{*}{$\begin{array}{l}b=0.25 \kappa=0.9 \\
g=0.75 \mu=0.1 \\
q=1.1 \alpha=0.1 \\
r=1.9 \sigma=0.25\end{array}$} & \multirow[t]{2}{*}{$P_{1}=0.302$} & $\begin{array}{l}P=0.1 \\
R_{0}(P)=1.992\end{array}$ & $\begin{array}{l}E_{0}=(0,0,0.208,0,0.208) \\
E_{1}=(0.109,0.101,0.0429,0.126,0.159)\end{array}$ \\
\hline & & $\begin{array}{l}P=0.4 \\
R_{0}(P)=0.809\end{array}$ & $\begin{array}{l}E_{0}=(0,0,0.033,0,0.131) \\
E_{1}: \text { Doesn't exist }\end{array}$ \\
\hline \multirow{2}{*}{$\begin{array}{l}b=1.25 \kappa=1 \\
g=0.05 \mu=0.15 \\
q=0.1 \alpha=0.3 \\
r=1.1 \sigma=0.4\end{array}$} & \multirow[t]{2}{*}{$P_{1}=0.391$} & $\begin{array}{l}P=0.38 \\
R_{0}(P)=1.016\end{array}$ & $\begin{array}{l}E_{0}=(0,0,0.228,0,0.577) \\
E_{1}=(0.005,0.001,0.223,0.003,0.575)\end{array}$ \\
\hline & & $\begin{array}{l}P=0.41 \\
R_{0}(P)=0.975\end{array}$ & $\begin{array}{l}E_{0}=(0,0,0.214,0,0.586) \\
E_{1}: \text { Doesn't exist }\end{array}$ \\
\hline
\end{tabular}

Table 1: Numerical simulation confirms the conjecture (3.3)

\section{References}

[1] R.M. Anderson, R.M. May, Infectious diseases of Humans: Dynamics and Control, Oxford University Press (2002).

[2] V. Andreasen, S.A. Lvin, J. Lin, Dynamics of annual influenza an epidemics with immuno-selection, Journal of Mathematical Biology, 46, No. 6(2003), 504-536. doi: http://dx.doi.org/10.1007/s00285-002-0186-2.

[3] M. BaniasadiMoghadam, H.M. MohammadiNezhad, O. RabieiMotlagh, Patterns of pandemic in a two satges SIRS models with a public health program, International journal of pure and applied mathematics, (2014).

[4] O. Diekmann, J.A.P Heesterbeek, Mathematical Epidemiology of Infectious Diseases: model building, analysis and interpretation, Wiley (2000). 
[5] O. Diekmann, J.A.P Heesterbeek, J. Metz, On the definition and computation of the basic reproduction ratio $R_{0}$ in models for infectious diseases in hetrogenous populations, Journal of Mathematical Biology, 28, No. 4(1990), 365-582. doi: http://dx.doi.org/10.1007/BF00178324.

[6] O. Diekmann, J.A.P Heesterbeek, M.G Roberts, The construction of the next-generation matrix for compartmental epidemic models, Journal of Royal Society Interface, 7, No. 47 (2010), 873-885. http://dx.doi.org/10.1098/rsif.2009.0386.

[7] H.W. Hethcote, The Mathematics of Infectious Diseases, SIAM Review, 42, No. 4(2002), 599-653. http://dx.doi.org/10.1137/s0036144500371907.

[8] W.O. Kermack, A.G. McKendrick, A contribution to the mathematical theory of epidemics, Proceeding of the royal society A, 115, No. 4(1927), 700-721. doi: http://dx.doi.org/10.1098/rspa.1927.0118.

[9] C.M. Kribs-Zaleta, J.X. Velasco-Hernandez, A simple vaccination model with multiple endemic states, Mathematical Biosciences, 164, No. 2(2000), 183-201. http://dx.doi.org/10.1016/s0025-5564(00)00003-1.

[10] S.A. Levin, B. Grenfell, A. Hastings, A.S. Perelson, Mathematical and computational challenges in population biology and ecosystem science, Science, 275, No. 5298(1997), 334-343. http://dx.doi.org/10.1126/science.275.5298.334.

[11] D.J. Murray, Mathematical Biology, Springer (1993).

[12] M. Safan, M. kretzschamer, K.P. Hadler, Vaccination based control of infections in SIRS models with reinfection: special reference to pertussis, Journal of Mathematical Biology, 67, No. 5(2013), 1083-1110. http://dx.doi.org/10.1007/s00285-012-0582-1. 
